\title{
Liquid Transport through Mosaic Membranes of Hydrophobic-Hydrophilic Microdomains: Pervaporation of Aqueous Ethanol Solutions through Bisphenol-A Polycarbonate-Polyoxyethylene Multiblock Copolymers
}

\author{
Hideaki TANISUGI and Tadao KoTAKA* \\ Department of Macromolecular Science, Faculty of Science, \\ Osaka University, Toyonaka, Osaka 560, Japan
}

(Received June 12, 1984)

\begin{abstract}
Pervaporation of ethanol/water mixtures at $25^{\circ} \mathrm{C}$ was examined on membranes of $(\mathrm{AB})_{n}$ type multiblock copolymers of bisphenol-A polycarbonate (PC) and polyoxyethylene (POE) with varying composition and block length. The membranes allowed preferential pervaporation of water over ethanol, and hence were effective for dehydration of the mixtures with high ethanol content. The rate of pervaporation was dominated by the solubility of the liquids to the membranes. Thus, the overall pervaporation rate was high for the swollen membranes. However, the permselectivity as judged by the separation factor $\alpha$, which is the ratio of the water versus ethanol content in the permeate and in the feed, was reduced by swelling. Thus, an optimum for better selectivity and yet a reasonably high rate of pervaporation was attained by the sample with $75 \mathrm{wt} \%$ PC content and long blocks for the azeotropic $(95.5 \%$ ethanol) mixture rather than by those with low PC content.
\end{abstract}

KEY WORDS Pervaporation / Separation of Ethanol and Water / Multiblock Copolymer / Microdomain Structure / Bisphenol-A PolycarbonatePolyoxyethylene Copolymer / Swelling / Pervaporation Rate / Separation Factor / Permselectivity /

The current increasing interest in biomass technology is demanding development of new efficient separation techniques applicable to aqueous solutions. ${ }^{1-4}$ Usually separation of components from aqueous solutions such as water and ethanol is a time- and energyconsuming process. In this respect, membrane technology is promising as energy saving processes of separation. ${ }^{3}$ Pervaporation is one of such membrane techniques particularly useful for azeotropic mixtures, which are hardly separable by conventional distillation. The technique is also convenient for evaluating the performance of permselective membranes.

In recent years, separation of aqueous alcohol solutions by selective permeation through polymer membranes was attempted by many researchers. ${ }^{5-17} \mathrm{~A}$ large portion of such studies was made using hydrophilic polymer membranes. ${ }^{5-11}$ Because hydrophilic membranes were lacking toughness due to excessive swelling in aqueous media, improvement of their toughness was attempted mostly by introducing crosslinks.

On the other hand, in accordance with current developments in varieties of polymer alloys, several other attempts were made to improve membranes, for example, by grafting hydrophilic segments onto hydrophobic hard backbones. ${ }^{12-15}$ Block copolymers, which also belong to a family of polymer alloys, are another candidates for high performance sepa-

* To whom correspondence should be addressed. 
ration membranes. ${ }^{16,17}$ They might exhibit additional advantages due to microphase-separated structures characteristic of the molecular architecture. In many block copolymer membranes, the existence of the intermixing phase appears to be imparting intriguing characteristics to the membranes. However, few workers described pervaporation characteristics of such block copolymer membranes in terms of their morphological features.

In this series of studies, we attempted to develop a method of separating aqueous ethanol mixtures by using multiblock copolymer membranes having mosaic structures of hydrophilic soft-domains and hydrophobic harddomains. The membranes tested were randomly-coupled bisphenol-A polycarbonate (PC) and polyoxyethylene (POE) multiblock copolymers of $(\mathrm{AB})_{n}$-type with varying composition and block length. In our previous articles, we described swelling behavior of these copolymers in water/ethanol mixtures, ${ }^{21}$ and also pervaporation of pure water and ethanol through these membranes, ${ }^{22}$ placing emphasis on the relation between these properties and the microdomain structures. We found that the swelling behavior of the copolymers in water/ethanol mixtures were quite different from that of the homopolymer membranes. ${ }^{21}$ We also found that depending on the nature of the permeant, its permeability was dominated either by diffusivity or solubility of the permeant into the membranes. Ethanol belongs to the former type, and water to the latter. ${ }^{22}$ The present paper is concerned with evaluation of permeation rate and permselectivity of these multiblock copolymer membranes for pervaporation of water/ethanol mixtures.

\section{EXPERIMENTAL}

\section{Materials}

The copolymer samples used were prepared by condensation of bisphenol-A (B) and polyoxyethylene glycol (POE) oligomer with
Table I. Characteristics of multiblock copolymers ${ }^{\mathrm{a}}$

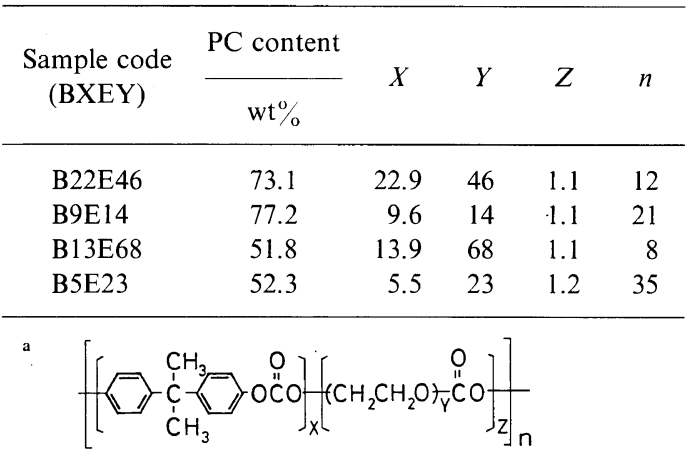

phosgene by the method of Goldberg. ${ }^{23}$ The details were described in our previous papers. ${ }^{18-22}$ Table I shows the characteristics of the four samples used in this study. The copolymers are coded as BXEY with $X$ and $Y$ denoting the average degree of polymerization of PC blocks and POE precursors, respectively. The values of $X$ and the average number $Z$ of POE precursors coupled to form individual POE blocks were calculated from the monomer feed-product composition by assuming random coupling of $\mathrm{B}$ and $\mathrm{EY}$ units.

The copolymer samples were cast into films of about $0.05 \mathrm{~mm}$ thick from $2 \mathrm{wt} \%$ chloroform solutions on a teflon sheet. The solvent was allowed to evaporate slowly over a week, and then dried under vacuum of $10^{-2}$ torr for two more days.

For the sample characterization, we carried out differential scanning calorimetry (DSC) with a Rigaku Denki Model 8055 DSC apparatus in a range from 170 to $570 \mathrm{~K}$ at a heating rate of $10 \mathrm{~K} \mathrm{~min}^{-1}$.

\section{Pervaporation}

In our previous study, ${ }^{21}$ we found that the swelling behavior of the BXEY polymers in water/ethanol mixtures significantly differed depending on whether they were conditioned by water or ethanol. Thus, we usually started a series of pervaporation tests first for a mixture of the lowest ethanol content (usually $20 \mathrm{wt} \%$ ). At the beginning, a freshly prepared mem- 
brane was equilibrated with a $20 \mathrm{wt} \%$ ethanol mixture. Then, the membrane was mounted on the pervaporation cell, and tested for the $20 \mathrm{wt} \%$ mixture. After finishing the first run, the feed was replaced by a mixture of next higher ethanol content, and the membrane was conditioned in situ with the mixture. After reaching the swelling equilibrium, the test was carried out for the second mixture. These procedures were repeated using solutions with increasing ethanol content, step-by-step, up to the azeotropic composition. In some cases, ethanol conditioned membranes were tested.

The apparatus and procedures for the pervaporation test were described in our previous paper. $^{22}$ All the pervaporation experiments were carried out at $25.0 \pm 0.1^{\circ} \mathrm{C}$. The downstream (vacuum) side of the membrane was kept at a pressure between $10^{-3}$ and $10^{-5}$ torr by a diffusion and rotary pumps with cold traps. The vacuum was monitored with a coldcathode ionization guage (Tokuda Seisakusho, Model PST-5). The feed was constantly stirred by a stirrer to prevent concentration polarization to occur. The permeate was collected using two cold traps chilled with liquid $\mathrm{N}_{2}$ alternately.

To determine the pervaporation rate $P$ $\left(\mathrm{g} \mathrm{cm}^{-1} \mathrm{~s}^{-1}\right)$ and the composition of the permeate, alliquots were collected at appropriate intervals. Whenever an alliquot (at least $0.5 \mathrm{~g}$ ) of permeate was collected, an alliquot was taken also from the feed to check the composition change in the feed.

The composition of the permeate and feed was analysed with a gas chromatograph (GC: Shimadzu Seisakusho, GC-4BM) equipped with a flame ionization detector (FID). The column was a $3 \mathrm{~m}$ glass column packed with $80-100$ mesh diatomite impregnated with 20 $\mathrm{wt} \%$ fatty acid (FFAP Chromosorb W; Wako Pure Chemicals Co.).

The chromatograms were calibrated with acetone/ethanol/water mixtures of known acetone/ethanol content. To do this, ethanol/ water mixtures of known composition were prepared as the initial standard mixtures. An exactly twice amount of acetone was added to each mixture. Then, the mixture was diluted with an appropriate amount of water so that we could detect adequate acetone and ethanol signals in the chromatogram. For a test, we injected $0.1 \mu \mathrm{L}$ of the sample to the GC several times, and determined the average ratio of the height of the ethanol and acetone peaks. The relative peak height was plotted against the water/ethanol composition of the initial mixture to make a calibration for the later analyses. The precision in determining ethanol content was in most cases within $\pm 0.3 \mathrm{wt} \%$.

In a typical experiment, a $200 \mathrm{~g}$ feed was charged, and the permeate of approximately 0.6 to $0.7 \mathrm{~g}$ was collected several times at $2 \mathrm{~h}$ interval to assure the steady state. The collected samples were subjected to the composition analysis as mentioned above. In a typical example for the azeotropic mixture with a B22E46 membrane, total $3.55 \mathrm{~g}$ permeate with the average $79 \mathrm{wt} \%$ ethanol content were recovered, while the composition of the feed changed from 95.5 to $96.5 \mathrm{wt} \%$ during the run. Thus, the mass balance was satisfied.

\section{Analysis of Pervaporation Data}

The selective permeation of an $A / B$ binary mixture can be conveniently expressed in terms of the separation factor $\alpha_{\mathrm{A}}$ defined as

$$
\alpha_{\mathrm{A}}=\left(Y_{\mathrm{A}} / Y_{\mathrm{B}}\right) /\left(X_{\mathrm{A}} / X_{\mathrm{B}}\right)
$$

where $X_{\mathrm{K}}$ and $Y_{\mathrm{K}}$ are the weight fraction of the species $K(K=A$ or $B)$ in the feed and permeate, respectively. ${ }^{4}$ Usually the preferentially permeating species is taken as the species $A$.

If the pervaporation proceeded ideally, the pervaporation rate of the component $K(K=A$ or $B$ ) should be given by ${ }^{4}$

$$
P_{\mathrm{k}}^{\mathrm{id}}=X_{\mathrm{K}} P_{\mathrm{K}}^{0} \quad(K=A \text { or } B)
$$

where $P_{\mathrm{K}}^{0}$ is the observed pervaporation rate of the pure liquid $K$ for the same membrane. Since the ideal pervaporation implicitly assumes an additivity of the flux of the com- 
ponents, the total ideal pervaporation rate $P^{\text {id }}$ should be

$$
P^{\text {id }}=P_{\mathrm{A}}^{\mathrm{id}}+P_{\mathrm{B}}^{\mathrm{id}}=X_{\mathrm{A}} P_{\mathrm{A}}^{0}+X_{\mathrm{B}} P_{\mathrm{B}}^{0}
$$

Huang et $a l^{5,6}$ introduced the permeation ratio $\theta$ and $\theta_{\mathbf{K}}$ as a measure of the deviation of the observed overall rate $P$ and the rate $P_{\mathrm{K}}^{\text {obs }}$ of the pure liquid $\mathrm{K}$ from the ideal rates $P^{\text {id }}$ and $P_{\mathrm{K}}^{\text {id }}$ as

$$
\begin{aligned}
& \theta=P / P^{\text {id }} \\
& \theta_{\mathrm{K}}=P_{\mathrm{K}}^{\text {obs }} / P_{\mathrm{K}}^{\text {id }}=Y_{\mathrm{K}} P / X_{\mathrm{K}} P_{\mathrm{K}}^{0}
\end{aligned}
$$

where $P_{\mathrm{K}}^{\mathrm{obs}}=Y_{\mathrm{K}} P$ is by definition the observed pervaporation rate of the component $K(=A$ or $B)$.

\section{RESULTS}

\section{Permselectivity}

First we compare the permselectivity of ethanol and water for the four copolymer samples tested. Figures 1 and 2 show plots of the ethanol content in the permeate $Y_{\mathrm{EtOH}}$ versus in the feed $X_{\mathrm{EtOH}}$ at $25^{\circ} \mathrm{C}$ for the membranes with 75 and $50 \mathrm{wt} \% \mathrm{PC}$ content, respectively. The separation factor $\alpha_{\text {water }}$ determined by eq 1 is also shown in the same figures. However, we could not carry out the pervaporation test on POE-rich B5E68 and B2E23 membranes, because they were usually highly swollen and lacking mechanical strength. ${ }^{21}$ These soft membranes at a highly swollen state were easily broken by applying vacuum for the pervaporation test.

In Figures 1 and 2, we notice that the observed data are always below the dashed curve representing the equilibrium vaporliquid composition diagram for water/ethanol mixtures at $25^{\circ} \mathrm{C}^{23}$ Thus, the main pervaporating species is water rather than ethanol, although at $25^{\circ} \mathrm{C}$ the former has the lower vapor pressure than the latter. This tendency was common for all the samples tested here, regardless of the composition and block length of the samples. Similar results were reported for pervaporation of water/ethanol mixtures
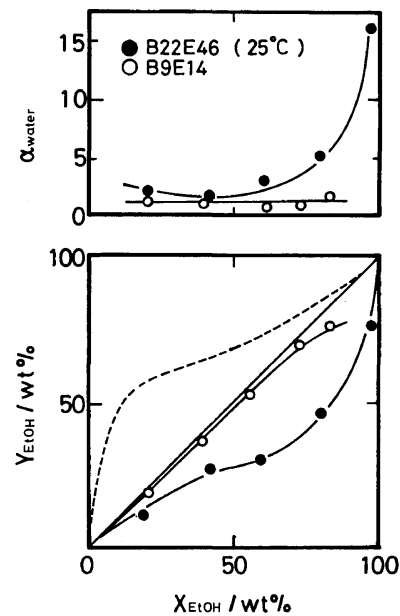

Figure 1. Ethanol composition $Y_{\mathrm{EtOH}}$ in the permeate and separation factor $\alpha_{\text {water }}$ as a function of ethanol composition $X_{\mathrm{EtOH}}$ in the feed for pervaporation at $25^{\circ} \mathrm{C}$ through $75 \mathrm{wt} \%$ PC containing membranes. The dashed line is the equilibrium liquid/vapor diagram for water/ ethanol mixtures at $25^{\circ} \mathrm{C}$.
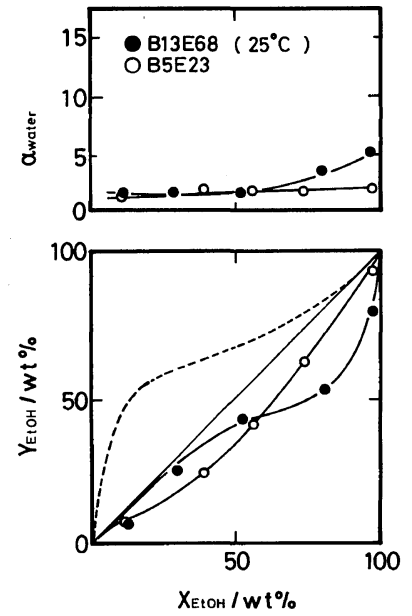

Figure 2. The same plots as in Figure 1 for $50 \mathrm{wt} \% \mathrm{PC}$ containing membranes.

through hydrophilic membranes. ${ }^{5-7,9-11}$

Comparing the two sets of membranes having the same PC content but different block length, we noticed that those with long blocks exhibited better performance over the other. For those with $75 \mathrm{wt} \%$ PC content, the values of $\alpha_{\text {water }}$ for the B9E14 membrane (having short blocks) were nearly 1 to 2 at most for the 
feed of any composition. On the other hand, $\alpha_{\text {water }}$ for the B22E46 membrane (having long blocks) increased significantly for the feed with increasing ethanol content over $50 \mathrm{wt} \%$ ethanol, and reached as high as roughly 16 for the azeotropic mixture. In the case of $50 \mathrm{wt} \%$ PC containing membranes, a better permselectivity was attained also by the sample with long blocks, although the increase in $\alpha_{\text {water }}$ was much less than that for $75 \mathrm{wt} \%$ PC containing membranes.

Comparing the two long block samples with different PC content, B22E46 and B13E68, we notice that the permselectivity is high for the former with $73 \mathrm{wt} \% \mathrm{PC}$ content rather than the latter with $52 \mathrm{wt} \% \mathrm{PC}$ content.

\section{Rate of Pervaporation}

Next, we examined the dependence of pervaporation rates on the $\mathrm{PC} / \mathrm{POE}$ composition and block length of the samples. Figures 3 and 4 show plots of pervaporation rate $P$ at $25^{\circ} \mathrm{C}$ versus the ethanol content in the feed for 75 and $50 \mathrm{wt} \%$ PC containing membranes. The dashed curves represent the ideal rate $P^{\text {id }}$ calculated by eq 3 from our previous data for the pure liquids. ${ }^{22}$ The figures also show the equilibrium degrees of swelling $q_{\mathrm{e}}$ (g-liquid/g dry membrane) of the same membranes in ethanol/water mixtures taken from our previous paper. ${ }^{21}$

As can be seen in Figures 3 and 4, for the PC-rich membranes the rate $P^{0}$ of pure ethanol was larger than that of pure water, while for the PC/POE 50/50 membranes the latter was larger than the former. Thus, the rate $P^{0}$ of pure water was much smaller for the former, B22E46 and B9E14, than for the latter, B13E68 and B5E23, while that of pure ethanol was larger for the latter than for the former. This tendency is probably reflecting the fact that the PC-rich membranes were swollen more in ethanol than in water, while the 50/50 membranes (and POE-rich membranes also) were swollen more in water than in ethanol. ${ }^{21,22}$

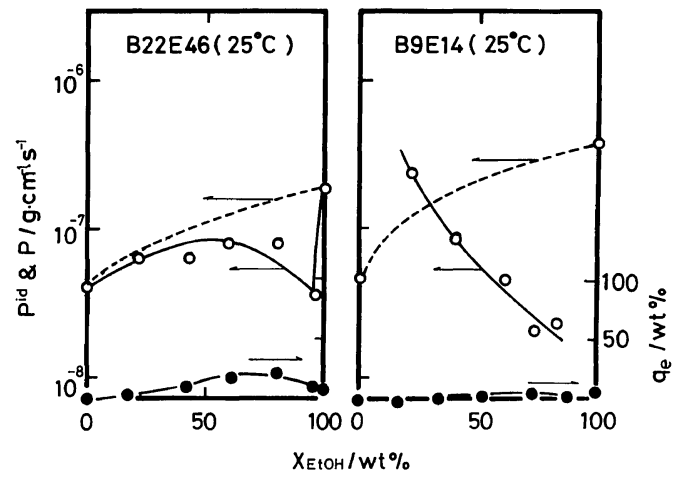

Figure 3. Observed (solid curves with open circles) and ideal (dashed curves) pervaporation rate as a function of ethanol composition $X_{\mathrm{E} 1 \mathrm{OH}}$ in the feed for pervaporation at $25^{\circ} \mathrm{C}$ through $75 \mathrm{wt} \%$ PC containing membranes. Equilibrium degree of swelling $q_{\mathrm{e}}(\mathrm{wt} \%)$ is also shown (solid curves with closed circles).

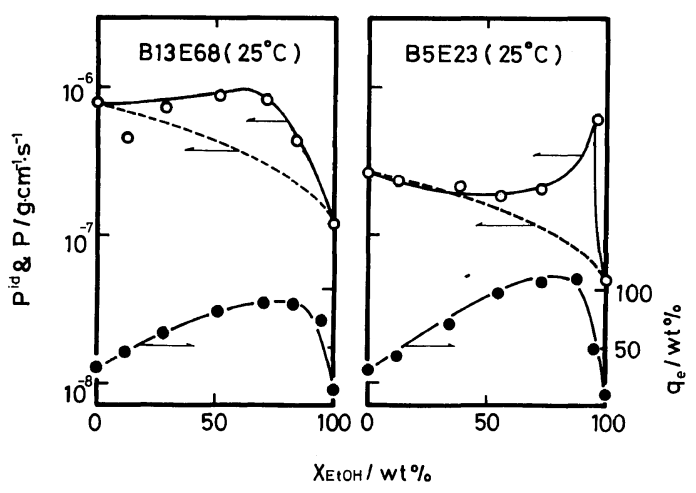

Figure 4. The same plots as in Figure 3 for $50 \mathrm{wt} \% \mathrm{PC}$ containing membranes.

In Figure 3, we see for the PC-rich membranes that the observed pervaporation rates $P$ were always smaller than the ideal rates $P^{\text {id }}$. The rate $P$ for $\mathrm{B} 22 \mathrm{E} 46$ exhibited a sharp minimum for the azeotropic mixture, while that for B9E14 steadily decreased with increasing ethanol content. The behavior of B9E14 was rather unusual in that $P$ for $20 \mathrm{wt} \%$ ethanol mixture was above the $P^{\text {id }}$ but decreased rapidly with increasing ethanol content in the mixtures, or by subjecting it to ethanol-rich mixtures.

On the other hand, for the $50 / 50$ membranes, $P$ exhibited a positive deviation from 


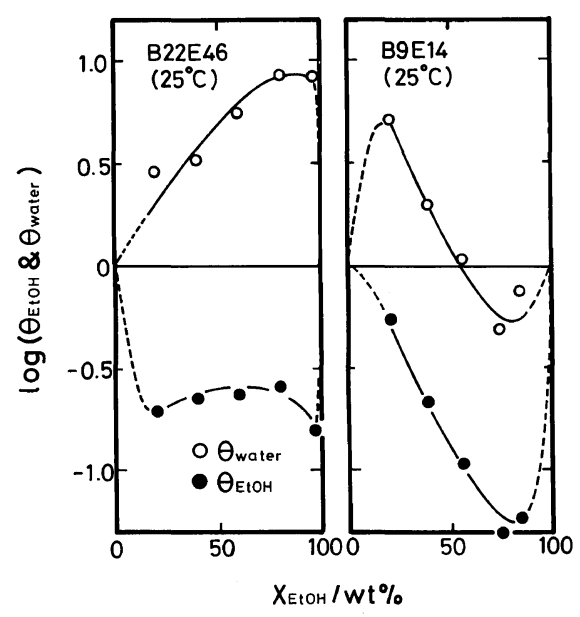

Figure 5. Plots of $\log$ (permeation ratio $\theta_{\text {water }}$ ) and $\log \left(\theta_{\mathrm{EtOH}}\right)$ versus ethanol composition $X_{\mathrm{EtOH}}$ in the feed for permeation at $25^{\circ} \mathrm{C}$ through $75 \mathrm{wt} \%$ PC containing membranes.

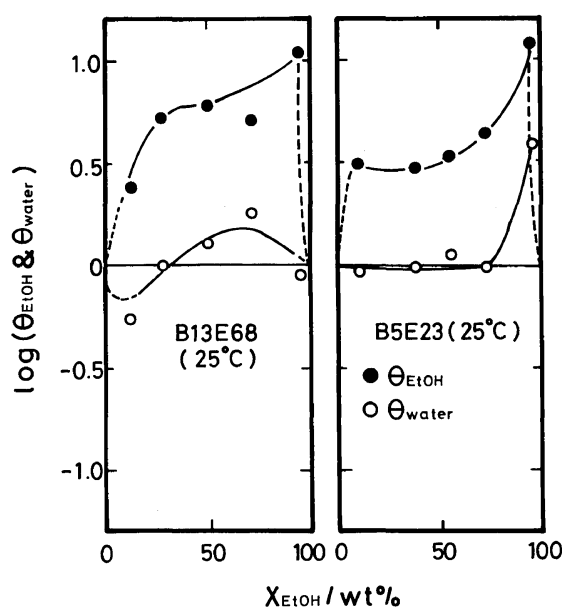

Figure 6. The same plots as in Figure 5 for $50 \mathrm{wt} \% \mathrm{PC}$ containing membranes.

the ideal behavior $P^{\text {id }}$, as can be seen in Figure 4. Particularly, that for B5E23 exhibited a sharp maximum for the azeotropic mixture.

The deviation of the observed behavior from the ideal one is also seen in the permeation ratios, $\theta_{\mathrm{EtOH}}$ and $\theta_{\text {water }}$, defined by eq 4 . Figures 5 and 6 show the plots of logarithm $\theta_{\mathrm{EtOH}}$ and $\theta_{\text {water }}$ versus feed composition $X_{\mathrm{EtOH}}$ for 75 and $50 \mathrm{wt} \%$ PC containing membranes, respectively. For the PC-rich membranes, $\theta_{\text {water }}$ was always larger than $\theta_{\mathrm{EtOH}}$. Particularly, for B22E46 $\theta_{\text {water }}$ was larger than unity, while $\theta_{\mathrm{EtOH}}$ was smaller than unity. Namely, the pervaporation of water was accelerated by absorbed ethanol, while that of ethanol was retarded by water. For B9E14 membranes, we notice a rather unusual dependence of the permeation ratios, $\theta_{\text {water }}$ and $\theta_{\mathrm{EtOH}}$, on the composition of the mixtures.

On the other hand, for the $50 \mathrm{wt} \% \mathrm{PC}$ containing membranes, $\theta_{\mathrm{EtOH}}$ is always larger than unity, while $\theta_{\text {water }}$ remained nearly constant close to unity except for a few cases. This means that the pervaporation of ethanol was always accelerated by water, but that of water was not affected by ethanol.

\section{Effect of Ethanol Conditioning}

In our previous work, ${ }^{21}$ we observed that ethanol conditioning of the BXEY membranes significantly affected their swelling behavior in water/ethanol mixtures, presumably through changes in microphase separated structures by conditioning. Ethanol conditioning appeared to promote microphase separation in $\mathrm{PC} / \mathrm{POE}$ intermixing domains and crystallization of $\mathrm{PC}$ blocks in the membranes. Thus, the degrees of swelling were reduced significantly, and their swelling behavior became similar to that of crosslinked POE membranes containing hydrophobic hard PC-rich domains. Thus, we examined the effect of ethanol conditioning on the pervaporation behavior of the two samples, B22E46 and B5E23, for comparison.

Figures 7 and 8 show the permselectivity and pervaporation rate for ethanol conditioned B22E46 and B5E23 membranes, respectively. Comparing the results on these unconditioned and ethanol-conditioned membranes, we notice for $\mathrm{B} 22 \mathrm{E} 46$ a slight reduction in the permselectivity, but for B5E23 a slight but appreciable improvement especially for the mixtures with high ethanol content. On the other hand, the observed pervaporation rates for both membranes were reduced significantly especially for the mixtures with high 


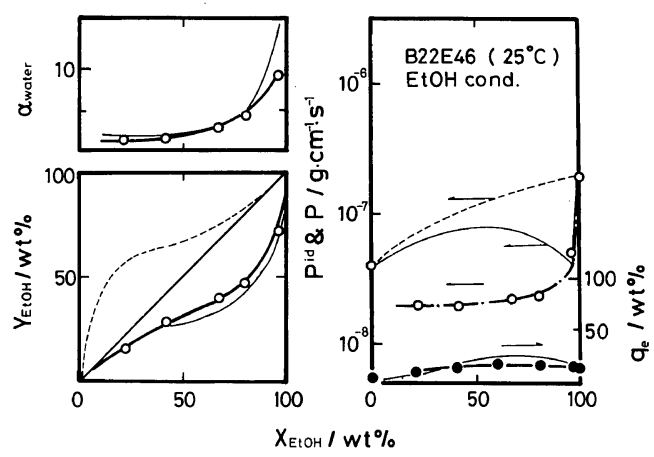

Figure 7. Separation factor $\alpha_{\text {water }}$, permeate composition $Y_{\mathrm{EtOH}}$, observed and ideal pervaporation rates, and equilibrium degree of swelling at $25^{\circ} \mathrm{C}$ for an ethanol conditioned B22E46 membrane. For comparison, the results for the unconditioned membrane are shown by thin lines.

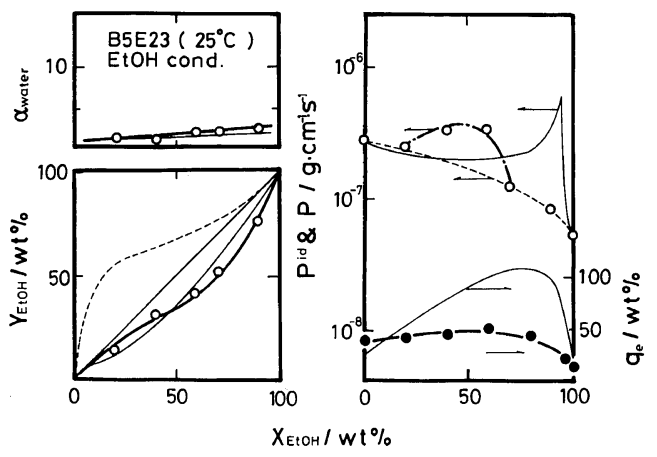

Figure 8. The same plots as in Figure 7 for an ethanol conditioned B5E23 membrane.

ethanol content.

\section{DISCUSSION}

The permeation of a liquid through a nonporous matrix is governed by two factors: The solubility and the diffusivity of the liquid through the matrix. A liquid with a high affinity dissolves well into the matrix, and the permeation is governed essentially by its solubility. On the other hand, a liquid with a low affinity hardly dissolves into the matrix, and the permeation is governed mainly by its diffusivity. However, once such a liquid is taken up, it can be transported rather rapidly through the matrix. For example, absorption and desorption of water in nonpolar polystyrene reach the equilibrium more rapidly than that in more polar styrene-acrylonitrile copolymer, although the maximum water regain by the former is much less than that by the latter. ${ }^{24}$ By comparing the solubility controlled and diffusivity controlled systems, it can be said that usually the overall-rate of pervaporation is higher for the former systems with the high affinity to the liquid.

Therefore, if we try to separate a mixture of two liquids such as water and a higher alcohol having a small mutual affinity, the strategy to be employed is rather simple: It is to select a membrane which has a high affinity to one of the liquid component but a poor affinity to the other. However, to separate two liquids having a high mutual affinity such as the present system, this strategy would not work. Even if the membrane has an uneven affinity to the component liquids, the absorbed component with the high affinity (to the membrane) would accelerate absorption of the other component. Pervaporation of such mixtures as water/ ethanol systems by a membrane consisting of mosaic domains having different affinities to the liquid components should be complicated due to the complex influence of the permeant/ matrix interactions.

From the results obtained in this study, we may conclude that through these BXEY membranes water permeates preferentially over ethanol. However, the mechanisms of the permselectivity appear to be different for the PC/POE 50/50 and PC-rich (75 $\mathrm{wt} \%$ ) membranes. Comparing Figures 3 and 4, we immediately notice that for the PC-rich membranes, the overall permeation rate exhibits a minimum for the mixture of nearly azeotropic composition, while for the $50 / 50$ membranes it exhibits a maximum for the same mixture.

On the basis of the dissolution-diffusion mechanism of the permeation of water through poly(vinyl alcohol) membranes and various alcohols through crosslinked natural rubber membranes, Paul et al. ${ }^{25-28}$ suggested 
that the permeation rate may depend on the viscosity, since the diffusivity is inversely proportional to the permeant viscosity. Hamaya and Yamada $^{29}$ reported on the pervaporation of $n$-butylamine/water mixtures through cellophane that the pervaporation rate exhibited a minimum at a certain composition of the mixture which has the maximum viscosity. They interpreted this result according to the permeant viscosity concept. However, this regime of Hamaya and Yamada ${ }^{29}$ on the pervaporation of liquid mixtures through singlephase membranes is obviously too simple to apply to our complex multiphase membranes.

The 50/50 membranes (especially with long blocks) are more hydrophilic than the PC-rich membranes, and swollen more in water than in ethanol. ${ }^{21}$ Therefore, the permeation of water should be governed essentially by the solubility of water into these $50 / 50$ membranes. ${ }^{20}$ However, we found that for the mixtures especially of high ethanol content, the degrees of swelling of these membranes became exceedingly large in comparison with the composition average for the pure liquids (see Figure 4). ${ }^{21}$ This increase in the solubility of ethanol to these $50 / 50$ membranes by the presence of water should accelerate permeation of ethanol and increase the overall rate $P$ over the ideal rate $P^{\text {id }}$, while that of water should be practically unaffected by coexisting ethanol (see Figure 6). These tendencies would bring about a minor improvement in the separation factor.

Although we did not test directly, we may deduce that since the POE-rich and other hydrophilic membranes as well are highly swellable in aqueous media, such membranes should exhibit a high pervaporation rate but a poor permselectivity for water/ethanol mixtures. By absorbing a small amount of water in the mixture, such membranes become capable of absorbing a large amount of ethanol, even if the membrane itself can not absorb much ethanol. Thus, the pervaporation of ethanol in the mixtures should be encouraged, and consequently the permselectivity can be reduced.
The membranes of low $(30 \%)$ PC content such as B5E68 and B2E23 were highly swellable in the mixtures especially of high ethanol content. $^{21}$ In fact, the B2E23 membrane was insoluble either in water or ethanol but soluble in the 70 to $80 \%$ ethanol mixtures. These membranes are obviously inadequate for separating ethanol and water, although the pervaporation rate could be very high.

On the other hand, the PC-rich membranes are hydrophobic and swollen more in ethanol rather than in water. ${ }^{21}$ Although they also exhibit a swelling maximum in the mixture of a certain ethanol content, the maximum uptake is much smaller than that of the $50 / 50$ membranes (compare Figures 3 and 4). In such membranes, absorption of water appears to be encouraged by coexisting ethanol, which probably acts as a plasticizer of hard PC domains and loosens the network structure having PCmicrodomains acting as physical crosslinks. ${ }^{18}$ Thus, permeation of water can be accelerated.

Our previous results ${ }^{22}$ showed that in the PC-rich membranes the pervaporation rate of pure ethanol is higher than that of pure water. We also observed that the average diffusion rate of pure ethanol is larger in PC-rich membranes rather than in POE rich membranes. These results imply that permeation of ethanol in the PC-rich membranes is governed by its dissolution and diffusion through PC-rich intermixing domains. In water/ethanol mixtures, polar POE domains containing absorbed water may have a higher affinity toward ethanol than the PC-rich domains, and thus the ethanol content in the PC-rich domains may be reduced, where ethanol diffuses rather rapidly. These factors may contribute to reduce the permeation ratio $\theta_{\mathrm{EtOH}}$. Thus, although the overall rate is low especially for the mixtures of high ethanol content, the permselectivity is rather high for these PC-rich membranes.

Comparing the behavior of the two samples having the same PC content but different block length, we notice that the separation factor $\alpha_{\text {water }}$ is better for the sample having long 
blocks. However, the reason for this difference appears to be different between the two systems. For the $75 \%$ PC content membranes, the solubility of the mixture in the B9E14 membrane having short blocks is almost the composition average of the pure liquids and is less than that in the B22E46 membrane having long blocks (see Figure 3). Thus, the permselectivity arises from the difference in the diffusivity of the two liquids. However, our previous results showed that the diffusivity of the two liquid in the B9E14 membrane is almost the same. ${ }^{22}$ For the $50 / 50$ membranes, the solubility to the B5E23 membrane having short blocks appears to be larger than that to the B13E68 membrane having long blocks (see Figure 4). Thus, the permselectivity may be controlled by the solubility and reduced by this higher swellability in the mixtures.

In connection with the effects of block lengths on the pervaporation through the BXEY membranes, we should probably comment the followings. Our previous results ${ }^{18,20}$ showed that the short-block membranes such as B2E4 and B3E9 exhibited the properties similar to a single-phase random copolymer: They exhibit a single sharp glass transition, ${ }^{18}$ and show a small maximum regain for sorption of water. ${ }^{20}$ Such short-block membranes also are unlikely to exhibit high performance as a separation membrane: The permselectivity could be high, but the permeation rate should be intolerably slow.

Turning our attention to ethanol conditioned membranes, we can comment the followings. Since microphase separation of the two components were promoted during ethanol-conditioning, these membranes should have acquired a more fully crystallized and microphase-separated structure and became less swellable either in water or ethanol. This tendency should certainly reduce the overall pervaporation rate of both $\mathrm{B} 22 \mathrm{E} 46$ and $\mathrm{B} 5 \mathrm{E} 23$ membranes (see Figures 7 and 8). For the former, the ethanol conditioning tended to reduce the degree of swelling slightly, and thus

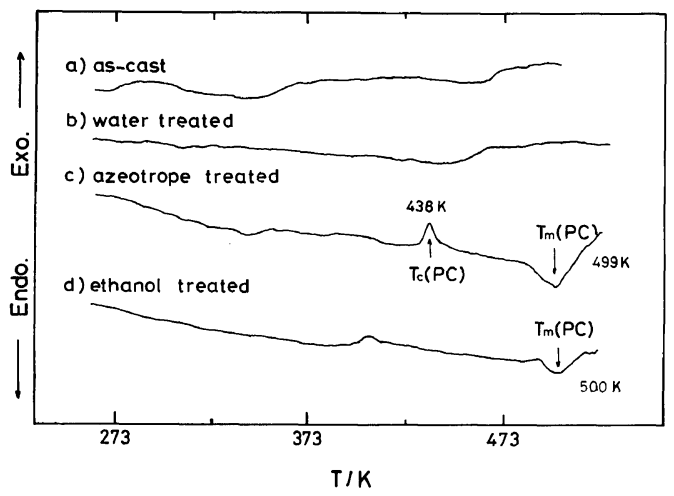

Figure 9. Changes in DSC curves of B9E14 by sample preconditioning: As-cast, water-conditioned, ethanol/water azeotropic mixture-conditioned and ethanol-conditioned membranes.

to reduce the pervaporation rate and permselectivity slightly (see Figure 7). On the other hand, for the latter, the decrease in $q_{\mathrm{e}}$ induced by the change in the microdomain structure is large for the mixtures of high ethanol content. This might imply the structural change reduced mainly ethanol uptake and the pervaporation rate of ethanol, thus improving slightly the permselectivity (see Figure 8).

Finally, we should probably comment on the rather unusual dependence on the ethanol content in the feed of the pervaporation rate and permselectivity exhibited by the B9E14 membrane. For this membrane, we noticed that the steady-state pervaporation was reached exceedingly slowly in comparison with other membranes, and that the reproducibility of the results was rather poor. These results suggest that some structural changes have taken place during the series of pervaporation experiments with varying feed composition.

Figure 9 shows DSC curves of the B9E14 membranes recovered at various stages of the pervaporation test. The membrane subjected to the test against an azeotropic mixture and pure ethanol exhibited a significant PCmelting peak at about $500 \mathrm{~K}$, which was absent in the as-cast membrane. During the pervaporation test against the mixtures with increasing ethanol content, further microphase sepa- 
ration and crystallization in the $\mathrm{PC}$ phase took place, and therefore the membrane became less swellable and tough. Thus, the rate of pervaporation was also significantly decreased with the sequence of experiments.

\section{CONCLUSION}

The performance of BXEY multiblock copolymer as permselective membranes was tested by pervaporation for water/ethanol mixtures. These membranes allowed extraction of water from the mixtures of high ethanol content. Among the four samples tested, the B22E46 membrane (with $75 \%$ PC content and relatively long blocks) exhibited the best performance in separation. The separation factor $\alpha_{\text {water }}$ reached as high as 16 for the azeotropic mixture, although the overall pervaporation rate was somewhat reduced.

The B13E68 membrane having 50/50 composition and long blocks exhibited the separation factor $\alpha_{\text {water }}$ of about 6 for the azeotropic mixture. However, its overall rate of pervaporation was about 10 times higher than that of the B22E46 membrane.

The BXEY membranes were good only for separation of the mixtures of high ethanol content. We are further seeking other membranes having the affinities different from the BXEY systems in the hope that we can develop a membrane system capable of extracting ethanol from the mixtures of low ethanol content.

Acknowledgements. The work was supported by the Ministry of Education, Science, and Culture (Mombusho) under grants 543026 (to TK) and 58350053 (to Professor Michio Kurata, Kyoto University, the coordinator), which are gratefully acknowledged.

\section{REFERENCES}

1. See, for example, U. Merten, Ed., "Desalination by Reverse Osmosis,” MIT Press, Cambridge, 1966.
2. H. P. Gregor and C. D. Gregor, Sci. Am., 239 (1), 88 (1978).

3. F. F. Hartline, Science, 206, 41 (1979).

4. S. Hwang and K. Kammermeyer, "Membrane in Separations," Techniques of Chemistry Series, Vol. VII, John Wiley \& Sons, New York, 1975.

5. R. Y. M. Huang and V. J. C. Lin, J. Appl. Polym. Sci., 12, 2615 (1968).

6. R. Y. M. Huang and N. R. Jarvis, J. Appl. Polym. Sci., 14, 2341 (1970).

7. E. G. Heisler, A. S. Hunter, J. Sciliano, and R. M. Treadway, Science, 124, 77 (1956).

8. E. Eustache and G. Histi, J. Membrane Sci., 8, 105 (1981).

9. E. Nagy, O. Borlai, and A. Ujhidy, J. Membrane Sci., 7, 109 (1980).

10. M. H. V. Mulder, J. O. Hendrikman, H. Hegeman, and C. A. Smolders, J. Membrane Sci., 16, 269 (1983).

11. A. Misra, F. W. Kroesser, and R. A. Shelden, $J$. Polym. Sci., Polym. Symp., No. 41, 145 (1973).

12. P. Aptel, J. Cuny, J. Joezfonvicz, G. Morel, and J. Neel, J. Appl. Polym. Sci., 16, 1061 (1972).

13. P. Aptel, N. Challard, J. Cuny, and J. Neel, J. Membrane Sci., 1, 271 (1976).

14. G. C. Tealdo, P. Cenepa, and S. Munari, J. Membrane Sci., 9, 191 (1981).

15. I. Itoh, Y. Ohkawa, K. Ishihara, and I. Shinohara, Polym. J., 15, 827 (1983).

16. H. Yasuda, C. E. Lamaze, and A. Schindler, $J$. Polym. Sci., A-2, 9, 1579 (1971).

17. P. Aptel and I. Cabasso, J. Appl. Polym. Sci., 25, 1969 (1980).

18. T. Suzuki and T. Kotaka, Macromolecules, 13, 1495 (1980).

19. T. Suzuki and T. Kotaka, Polym. J., 15, 15 (1983).

20. T. Suzuki, H. Chihara, and T. Kotaka, Polym. J., 16, 129 (1984).

21. H. Tanisugi, H. Ohnuma, and T. Kotaka, Polym. J., 16, 633 (1984).

22. H. Tanisugi and T. Kotaka, Polym. J., 16, 909 (1984).

23. C. Kux, Ed., "Landolt-Börnstein Zahlenwerte und Functionene aus Physik, Chemie, Astoronomie, Geophysik und Technik," Band II, Teil 2, SpringerVerlag, Berlin, 1960.

24. T. Suzuki, K. Adachi, and T. Kotaka, Polym. J., 13, 385 (1981).

25. D. R. Paul and O. M. Ebra-Lima, J. Appl. Polym. Sci., 14, 2201 (1970); ibid., 15, 2199 (1971).

26. D. R. Paul, J. Polym. Sci., 16, 771 (1972).

27. D. R. Paul and D. H. Carrnza, J. Polym. Sci., Polym. Symp., No. 41, 69 (1973).

28. O. M. Ebra-Lima and D. R. Paul, J. Appl. Polym. Sci., 19, 1381 (1975).

29. T. Hamaya and S. Yamada, Kobunshi Ronbunshu, 36, 419 (1979); ibid., 37, 65 (1980). 